\title{
Clustering and layering of In adatoms on low and high index silicon surfaces: A comparative study
}

\author{
Jithesh Kuyyalil ${ }^{\text {b }}$, Govind ${ }^{\text {a }}$, Mahesh Kumar ${ }^{\text {a }}$, S.M. Shivaprasad ${ }^{\text {b,* }}$ \\ a Surface Physics and Nanostructures Group, National Physical Laboratory, Dr. K.S. Krishnan Marg, New Delhi, 110012, India \\ b Jawaharlal Nehru Center for Advanced Scientific Research, Jakkur, Bangalore, 560064, India
}

\begin{abstract}
A B S T R A C T
Using the morphological differences of low and high index surfaces as templates for metal growth, several low dimensional overlayer structures with novel structural and electronic properties can be formed. We present here a first report on submonolayer adsorption and residual thermal desorption studies of In adatoms on reconstructed high index Si (5 5 12) $-2 \times 1$ surface and compare it with the observations on planar $\mathrm{Si}$ (111) $-7 \times 7$ surface. The study is done by using in-situ Ultra High Vacuum surface sensitive probes like Auger Electron Spectroscopy (AES) and Low Energy Electron Diffraction (LEED). These conventional wide area techniques provide an understanding of atomistic issues involved in the evolution of the interface. We have observed an anomalous growth mode during adsorption at room temperature (RT) above 2ML, which includes adatom layering and clustering on Si (111) surface. This is also manifested during the desorption experiments on both surfaces, and the subtle differences on the two surfaces are discussed. The observation of LEED pattern during the adsorption process shows formation of different superstructural phases on Si (111) $-7 \times 7$ surface. On Si (5 5 12) $2 \times 1$ surface we observe the sequential $2 \times(225), 2 \times(337)$ and $2 \times$ (113) facet formation during adsorption/desorption, which include quasi 1D-nanowire/chain structures. A combination of lattice strain effects, presence of step-edge barrier and quantum size effects are employed to speculate the differences in adsorption and desorption.
\end{abstract}

\section{Introduction}

Under thermodynamically driven conditions one expects a metal/ semiconductor epitaxial growth to follow one of the three main growth modes viz. Frank Van der Merwe, Stranski Krastanov or Volmer Weber [1]. It is also very well known that these growth conditions can be overridden by kinetic parameters which can result in the formation of different superstructural and meta stable phases, which cannot be thermodynamically achieved $[2,3]$. Recently, it is reported that in the ultrathin film regime, because of the prominence of quantum effects under certain kinetic conditions, the quantum size induced energy minima itself can control growth morphology leading to the formation of well ordered nano-islands [4,5]. Also, there are reports which show the influence of the step-edge diffusion barriers on islands, leading to the anomalies in the growth processes [6,7].

Epitaxial growth has been of great interest over the last three decades because of the possibility it offers in the fabrication of ordered nanostructures with tailored properties [8]. Studies of metals on semiconductor surfaces has attracted the attention of researchers as it was shown that stable surface reconstructions act as templates for the growth of self-assembled nanostructures. Metal adsorption on $\mathrm{Si}(111)-7 \times 7$ surfaces owing to its corner holes and faulted and unfaulted halves of the unit cell, has promoted the formation of magic clusters, nanowires etc [9]. Of late, there are several studies of metal adsorption on the high index Si (55 12) $2 \times 1$ surface, which have enabled researchers to grow low dimensional structures along the channels $[10,11]$, which has also led to the observation of several quantum phenomena $[12,13]$.

Si (5 5 12) $2 \times 1$ surface is a very interesting surface with its inherent trenched morphology. These faceted grooves provide researchers an opportunity to grow 1D nanostructures with unprecedented assembly control. These systems display novel structural and electronic properties and also enable the understanding of low dimensional physics of metallic overlayers. This indeed was proved by several researchers $[10,11]$, immediately after it was first discovered by Baski et al [14]. The (5512) surface lies about $30.5^{\circ}$ below the (001) plane as shown in Fig. 1 , which is a cross-sectional schematic view of the bulk silicon cut perpendicular to the $[66 \overline{5}$ ] direction $[14,15]$. The $(5512)$ unit cell is composed of two units of (337) and one unit of (225) facets, and a $2 \times 1$ reconstruction of the bulk truncated structure minimizes the energy by reducing the number of dangling bonds from 48 to 23 [15]. Detailed structural analysis has shown that the $2 \times 1$ reconstruction consists of a (337) unit with a dimer-adatom row, another (337) unit with a tetramer row and a (225) unit with a dimer-adatom and tetramer row. 


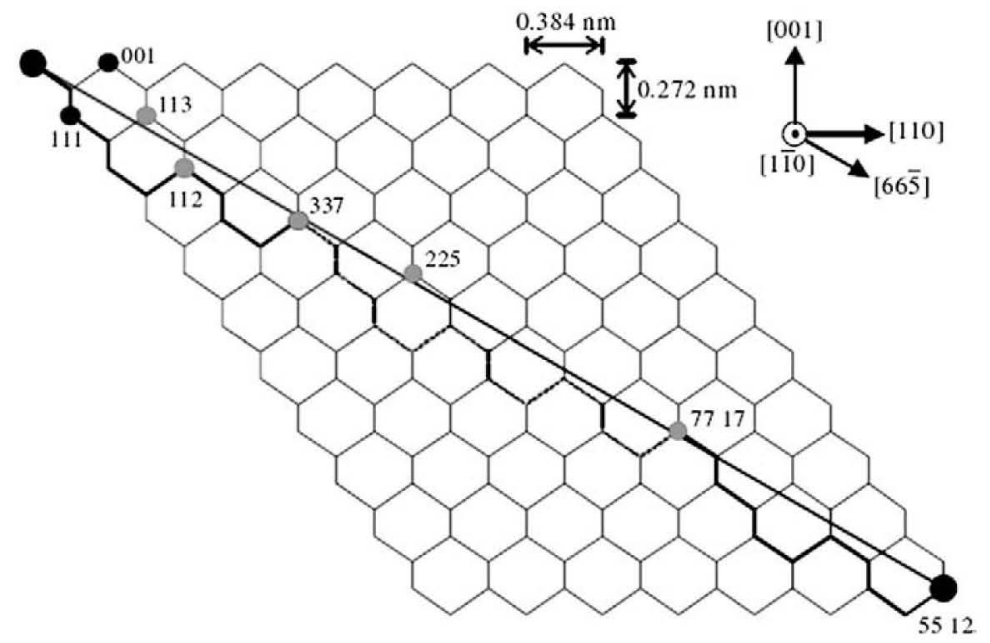

Fig. 1. A cross-sectional view of the bulk Si cut perpendicular to the (66 $\overline{5})$ direction showing the $\mathrm{Si}(5512)$ surface which lies about $30.5^{\circ} \mathrm{C}$ from the horizontal (001) plane. The solid circles mark unit cells of number of bulk-terminated orientations. Also shown are the different facets (113), (225), (337), (112) and (7 7 17).

As shown in the figure the surface can also be decomposed into different combinations of (225), (337), (112), (113) and (7 7 17). It is also reported that under appropriate kinetic conditions, metal adsorption can lead to the conversion of the (5 512 ) surface into different facets [26].

In this paper we present the first report on the adsorption/desorption studies of In on high index Si (5 5 12) $-2 \times 1$ surface and compare the results with the behavior of In adatoms on the $\mathrm{Si}(111)-7 \times 7$ surface. In/ $\mathrm{Si}(111)$ system is well studied in the literature and presents several surface phases and the growth follows the Stranski-Krastanov growth mode [16-18]. In this report we have used in-situ Auger Electron Spectroscopy (AES), Low Energy Electron Diffraction and Residual Thermal Desorption to study the growth of In on the low and high index silicon surfaces. AES provides an effective way of probing the evolution of the surface morphology of the adsorbate in sub-monolayer regime during the adsorption and residual thermal desorption process. Because of the finite inelastic mean free path of the electrons, the Auger uptake curve depicts characteristic features for different growth mode. For a given number of adsorbed atoms, the Auger intensity ratio will be lower for agglomerate (island) than when they form a flat layer, because of the attenuation of the Auger signals from the atoms inside the islands and due to the exposed substrate surface. We have used this strong feature of Auger Electron Spectroscopy to study the layering and clustering effects in the In/Si (111) system. We have previously reported the results on the adsorption studies of In on Si (111) $-7 \times 7$ surface [19]. The room temperature adsorption of In on the $\mathrm{Si}$ (111) surface shows an anomalous growth mode above $2 \mathrm{ML}$ where we observe layering and clustering of In adatoms. On Si ( 5512 ) surface we observe the formation of a quasi $1 \mathrm{D}$ chains. This behavior is re-manifested in the residual thermal desorption studies. We discuss these issues in the light of recent literature on quantum size effect in ultrathin films and the presence of step-edge barrier in the growing clusters.

\section{Experimental}

All the experiments are performed in situ in an Ultra High Vacuum System (Varian VT-112) with a base pressure of $3 \times 10^{-11}$ Torr. The chamber is equipped with a four grid LEED optics for probing the structure and a Cylindrical Mirror Analyzer (0.18\% resolution) with a concentric 0-10 kV electron gun for AES measurements. Modified Shiraki process $[20,21]$ is employed to chemically clean the Si substrate before inserting it in vacuum onto a high precision manipulator. The substrate is held by Ta clamps and heated resistively. Temperature measurements are made with an error of $\pm 8{ }^{\circ} \mathrm{C}$ by using a W-Re (5-25\%) thermocouple, calibrated by an optical pyrometer. The sample is annealed at $700{ }^{\circ} \mathrm{C}$ for several hours and then flashed $2-3$ times at $1150{ }^{\circ} \mathrm{C}$ for a few seconds and cooled gradually to room temperature to obtain an atomically clean $\mathrm{Si}(111)-7 \times 7$ and $\mathrm{Si}(5 \quad 5 \quad 12)-2 \times 1$ reconstructed surfaces, with characteristic AES spectra and LEED pattern. A homemade Ta Knudsen cell is used for In evaporation at desired flux rates and coverages by controlling the current to the cell. One monolayer (ML) coverage is defined as the density of a single (111) layer of bulk truncated Si i.e. $7.8 \times 10^{14}$ atoms per $\mathrm{cm}^{2}$. Thorough degassing ensures that the base pressure rises to a maximum of $2 \times 10^{-10}$ Torr even during extended In deposition and annealing. Low temperature annealing was accomplished by radiative heating by a proximal Ta filament up to $150{ }^{\circ} \mathrm{C}$ and by resistive heating of the sample to the desired higher temperature. The sample is held at the particular temperature for $1 \mathrm{~min}$ each for residual thermal desorption studies and cooled to RT before making AES and LEED measurements. All the data is acquired digitally and analyzed suitably to understand the kinetics of adsorption and the dynamics of desorption.

\section{Results and discussion}

Fig. 2a and d shows the LEED pattern of the clean $\operatorname{Si}(111)-7 \times 7$ and $\mathrm{Si}$ ( 55 12) $2 \times 1$ surface reconstructions respectively. Also shown in Fig. 2 are the line scans of the different LEED patterns of Si (5 5 12) surface along the $[66 \overline{5}]$ direction. The $7 \times 7$ reconstruction, which has 19 dangling bonds per unit cell, is well described by the famous DAS (dimer-adatom-stacking fault) model [22], with characteristic six fractional order spots between the sharp bulk spots while the high index Si (5 5 12) surface, has 16 fractional spots contributed by the (337) and (225) facets that it comprises of. Of the 23 dangling bonds of the (5 5 12) six, nine and eight originate from the dimer (337), (225) and tetramer (337) units respectively [15]. The faint streak, which goes parallel to the [ $66 \overline{5}$ ] direction, marks the $x 2$ periodicity of the reconstruction along the [1 10] direction [11]. From the analysis of intensity line scans along the [66 $\overline{5}$ ] direction, the distance between two consecutive spots in reciprocal space is found to be $1.17 \mathrm{~nm}^{-1}$ which corresponds to $5.35 \mathrm{~nm}$ in real space [11]. The spacing along the [ $\overline{1} 10]$ direction $8.15 \mathrm{~nm}^{-1}$ (real space distance of $0.77 \mathrm{~nm}$ ) is the double periodicity of the unreconstructed surface along this direction.

We carried out adsorption experiments to understand the growth mode of In on the two surfaces with different substrate structures. Fig. 3 shows the uptake curve for the adsorption of In on the $\mathrm{Si}(111)-7 \times 7$ and $\mathrm{Si}$ ( 55 12) $-2 \times 1$ surface kept at room temperature (RT). Reports in literature show that the growth of In on the Si (111) $-7 \times 7$ surface proceeds in the Stranski-Krastanov mode, with 3D islands on an uniform pseudomorphic In layer up to 2ML [16,23]. In Fig. 3 the ratio of Auger signals of In MNN ( $404 \mathrm{eV}$ ) to the Si LVV (92 eV) transitions is plotted against the deposition time. The overall trend of growth of In on the two surfaces shows a linear segment up to about ten minutes of adsorption which we attribute to the completion of 1ML [19] and the second 10 min segment is linear with a higher slope. However, the uptake curve of the two surfaces (111) and (5 512) show differences in both the segments of the curve. To accurately determine the break point, we have used the SSQ (sum of squares of errors) method [24] from the literature, where one calculates the errors in the slopes of the linear fit to the points around which a break is suspected. The calculated SSQ is plotted as a dashed curve along with the uptake curve in Fig. 3, whose minima corresponds to the break point at $10 \mathrm{~min}$ of adsorption. On Si (111) we take this point to correspond to $1 \mathrm{ML}$ and thus the flux rate is determined to be 0.1ML per minute [24].

The interpretation of LEED for room temperature adsorption becomes difficult owing to the low intensity of the fractional order spots and slight disorder in the surface phases formed. However, intensity line scans of the observed LEED pattern changes. On the $\mathrm{Si}(111)-7 \times 7$ surface with 0.5ML adsorption of In, the fractional order spots becomes weak (Fig. 2 (b)) and disappear at $2 \mathrm{ML}$, resulting in a clear $(1 \times 1)$ pattern (Fig. $2(\mathrm{c})$ ). On the $\mathrm{Si}$ ( 55 12) $-2 \times 1$ surface, when $0.2 \mathrm{ML}$ of In is adsorbed, the weak streak along the $[\overline{1} 10]$ direction vanishes, showing that the $\mathrm{x} 2$ periodicity 

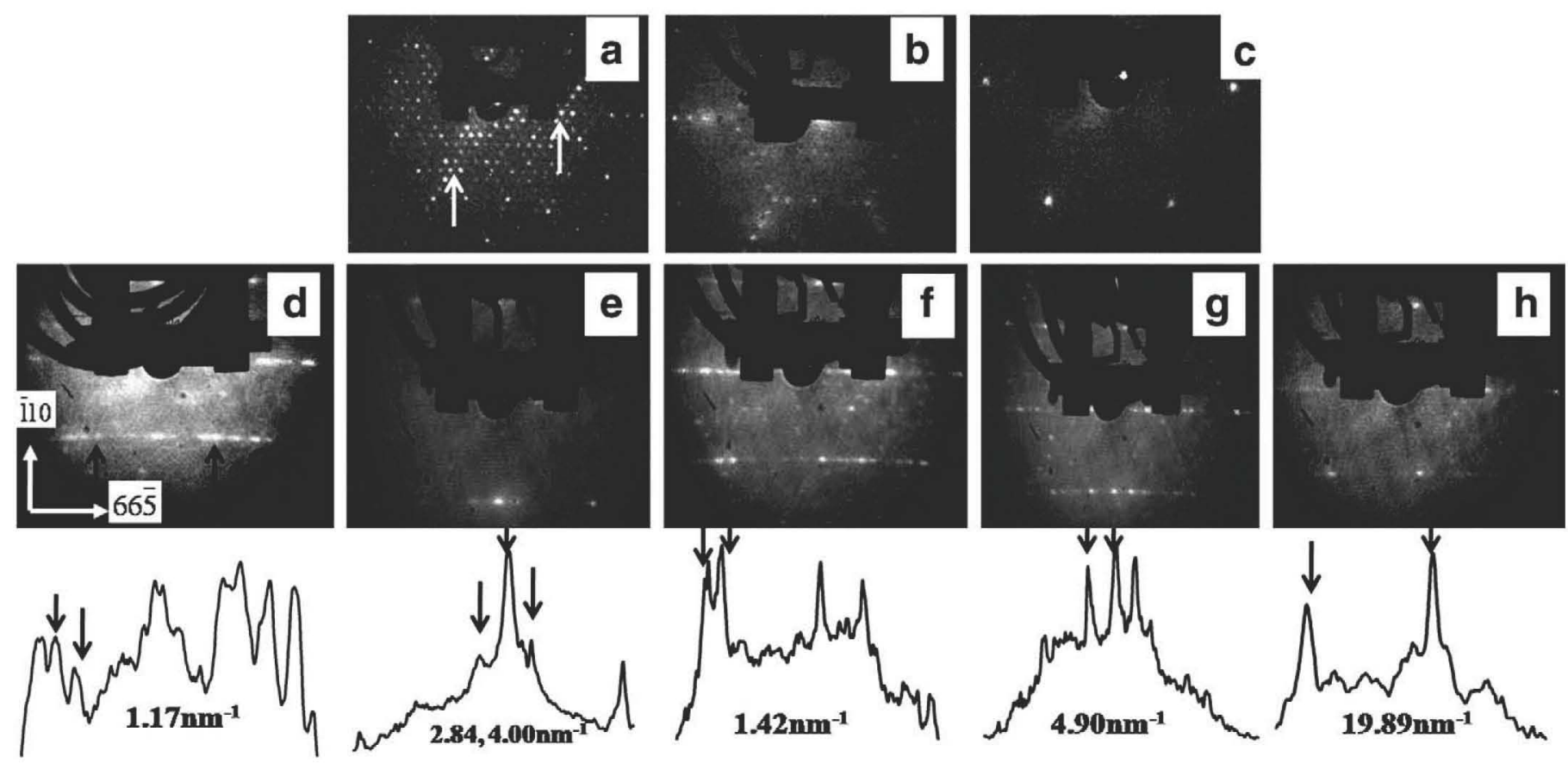

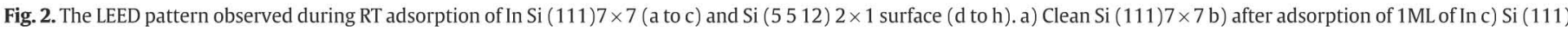

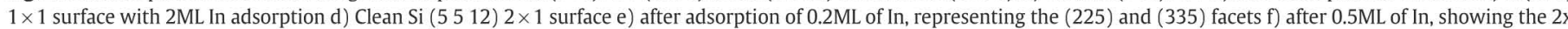

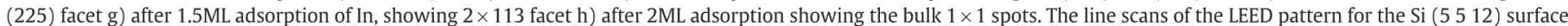
taken along [66 5] direction are shown at the bottom panel.

of the reconstruction is lifted along the [110] direction, resulting in the bulk truncated (5 512) surface. The lattice separation becomes $0.384 \mathrm{~nm}$ along this direction [11] and the line scan along the [66 5] direction (Fig. 3e) shows the unit cell periodicity of two facets viz. (225) and (337). Thus, we infer that the two differently structured (337) units undergo structural transformation to become a single (337) unit. The fractional order spots along the [66 5] direction diminish in intensity with adsorption up to 0.5ML. At around 0.5ML of adsorption, the intensity line scan along the [665] direction shows the formation of a well ordered In induced $2 x$ (225) facet as shown in Fig. $2 \mathrm{f}$. Previous studies on $\mathrm{Au}$ and $\mathrm{Sb}$ on Si (5 5 12) surface have shown that these facets consist of adsorbate induced quasi 1D (one dimensional) chain like features [11,25]. Though the exact structure and electronic properties of these facets are known for other systems [25], there are hardly any for In. The interchain separation can be measured by calculating the separation of the spots in the LEED

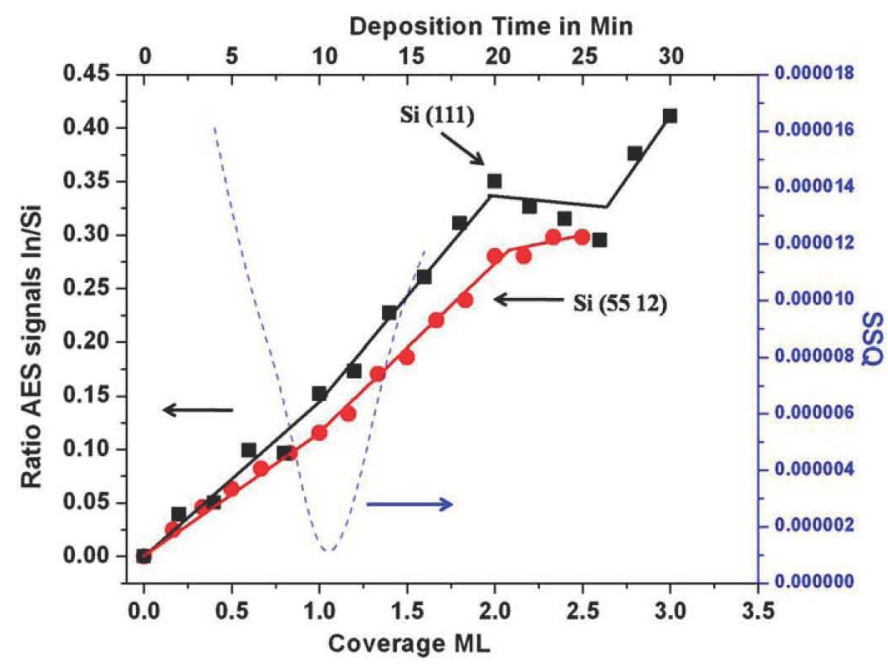

Fig. 3. The Auger uptake curve for RT In adsorption. The corresponding deposition time is shown in the top $x$-axis. The right $y$-axis represents the SSQ calculated to find the break in the uptake curve, which is shown as dashed curve in the figure. pattern which is found to be $1.42 \mathrm{~nm}^{-1}$ in reciprocal space, which corresponds to a real space distance of $4.4 \mathrm{~nm}$. When the coverage is increased, we observe weakening of the fractional order spots.

For coverages greater than 1ML the slope of the second part of the segment of the uptake curve is higher, as shown in Fig. 3. This increase in the slope of the uptake curve shows the formation of second layer of In on the surface. At about a coverage of $1.5 \mathrm{ML}$, we observe LEED fractional order spots which correspond to $2 x$ (113) facet shown in Fig. 2(g), which is previously reported to be consisting of $1 \mathrm{D}$ nanochains [26]. The analysis of the line scan along [66 5] gives a reciprocal lattice spacing of $4.90 \mathrm{~nm}^{-1}$, which is equivalent to $1.28 \mathrm{~nm}$. When the coverage becomes 2ML the uptake curve saturates, indicating the formation of 3D islands on the surface. This behavior is qualitatively similar for both the surfaces and the growth proceeds in a similar fashion. The intensity of fractional order spots becomes very weak at this coverage and we could only observe bulk spots in the LEED pattern on both the surfaces as shown in Fig. 2(c) and (h). Further deposition of Indium results in the rise of intensity of AES ratio for $\mathrm{Si}$ (111) surface which is attributed to the layering of the islands. But we could not observe similar behavior on the Si (5 5 12) surface, which shows only the saturation of intensity ratio. Also we observe from Fig. 3 some differences between the uptake curves on the two surfaces. The ratio of AES intensities for the two surfaces, Si (111) $7 \times 7$ and $\mathrm{Si}$ ( 55 12) $-2 \times 1$, deviate less below $1 \mathrm{ML}$, but is greater for higher coverages, due to the structural differences of the two surfaces. Thus overall growth mode is that of Stranski-Krastanov growth mode with anomalous clustering and layering of In adatoms on the Si (111) surface. A schematic of the general morphology of the observed growth mode on the two surfaces is shown in Fig. 4a assuming a final coverage of 3ML on the surface. The schematic also shows the desorption of 3ML In system at different temperatures which will be described while explaining the thermal desorption results.

In the recent literature there is a lot of interest regarding the quantum size effects in metallic thin overlayer formation [27]. Researchers have observed that under favorable conditions, assisted by the kinetics of the growth, the quantum size effect energy can find a certain minima leading to certain metastable phases which have preferred island sizes [28]. In the present experiments for the observed 


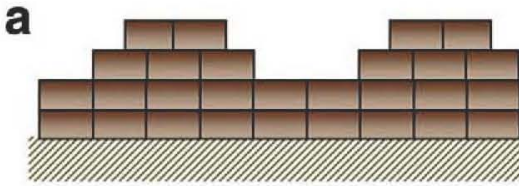

(3) $3 \mathrm{ML}$

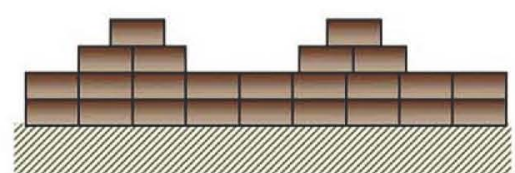

(4) $2.5 \mathrm{ML}$

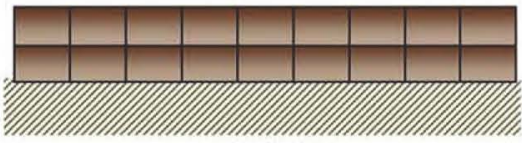

(2) $2 \mathrm{ML}$

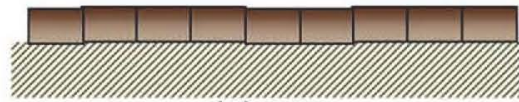

(1) $1 \mathrm{ML}$

Adsorption

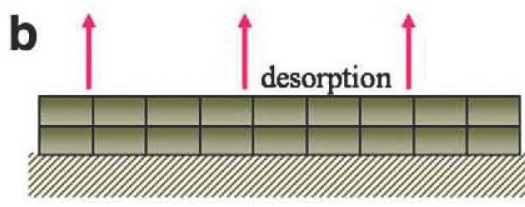

$>500^{\circ} \mathrm{C}$

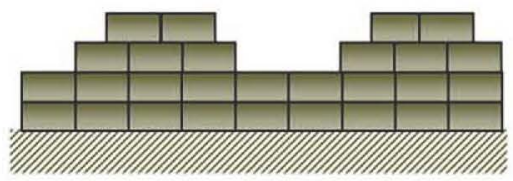

$500^{\circ} \mathrm{C}$

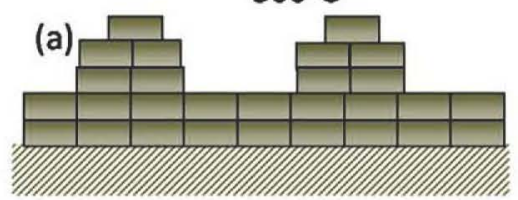

$350^{\circ} \mathrm{C}$

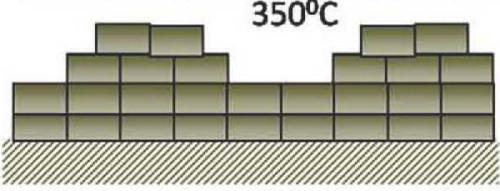

RT

Desorption

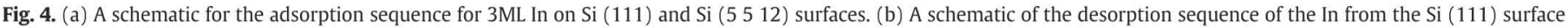
assuming an initial coverage of $3 \mathrm{ML}$.

growth morphology, we speculate that the quantum size effect may be dominating during the growth leading to certain island sizes. As the coverage on the surface increases, the confinement effect induced stability reduces and the size of the islands becomes large and consequently agglomerate to form layers. We will refer back to these observations while describing the residual thermal desorption results. As previously demonstrated (for other metals) by our group [11] and others [25], our present experiments have shown that the trenches on the $\mathrm{Si}$ (5 5 12) surface can be used as templates to grow 1D In nanochains. We note that the major structural change in the LEED pattern on the Si (111) surface takes place at $2 \mathrm{ML}$ i.e. the conversion $7 \times 7$ reconstruction into $1 \times 1$ and on the $\mathrm{Si}(5512)$ surface we observe conversion of facets at around $1 \mathrm{ML}$. This will be later corroborated with the desorption process.

The thermal stability of the system is studied by residual thermal desorption experiments where the system is subjected to annealing at different temperatures for fixed times, and the consequent AES and LEED results are shown in Figs. 5 and 6 respectively. Fig. 5 shows the variation of the ratio of the In (404 eV) MNN peak to the silicon (92 eV) LVV peak with annealing temperature for both $\mathrm{Si}$ (111) and Si (5 5 12) surfaces. For comparison, we have presented two representative data from $\mathrm{Si}$ (111) and $\mathrm{Si}(55$ 12) surfaces. For $\mathrm{Si}$ (111) surface the initial coverage on the surface is about 3.5ML consisting of two flat layers of In with islands above. For the $\mathrm{Si}(5512)$ surface initial coverage on the surface is 2.2ML with two flat In layers plus islands on top. This description of the initial morphology follows from the interpretation of the observed uptake curve. The overall trend of the two desorption curves are the same, but with subtle differences. To facilitate discussion, we divide the desorption curve into five different regions, marked as [1], [2]... [5] in Fig. 5. Both the curves show an initial fall in the intensity ratio up to a temperature of $300{ }^{\circ} \mathrm{C}$ (region [1]), 2nd region from 300 to $450{ }^{\circ} \mathrm{C}$, where the intensity remains constant, followed by a rise in the intensity ratio at a temperature of $450{ }^{\circ} \mathrm{C}$ (region [3]) and final steep fall in the ratio with temperature (region [4] \& region [5]). These observations can be explained as follows: When the adsorbed adlayer is subjected to annealing, there is an initial fall in the intensity ratio due to the agglomeration of the adatom into larger clusters, which may cost energy because of the creation of new surfaces. This is compensated by the strain relaxation in the as-adsorbed overlayer, due to the $14.8 \%$ lattice mismatch between surface and overlayer [23]. The electron diffraction shows the $1 \times 1$ pattern from the overlayer as shown in Figs. $2 \mathrm{c}$ and $6 \mathrm{e}$, which becomes sharper with annealing. The Arrhenius activation energy for agglomeration is calculated to be $0.08 \mathrm{eV}$ on the (111) surface and $0.11 \mathrm{eV}$ on (5 5 12) surface [29].

The flat portion of the desorption curve (region [2] in Fig. 5) shows that the morphology on the surface remains essentially the same in this temperature range. Compared to the previous region, on the $\mathrm{Si}$ (5 512 )

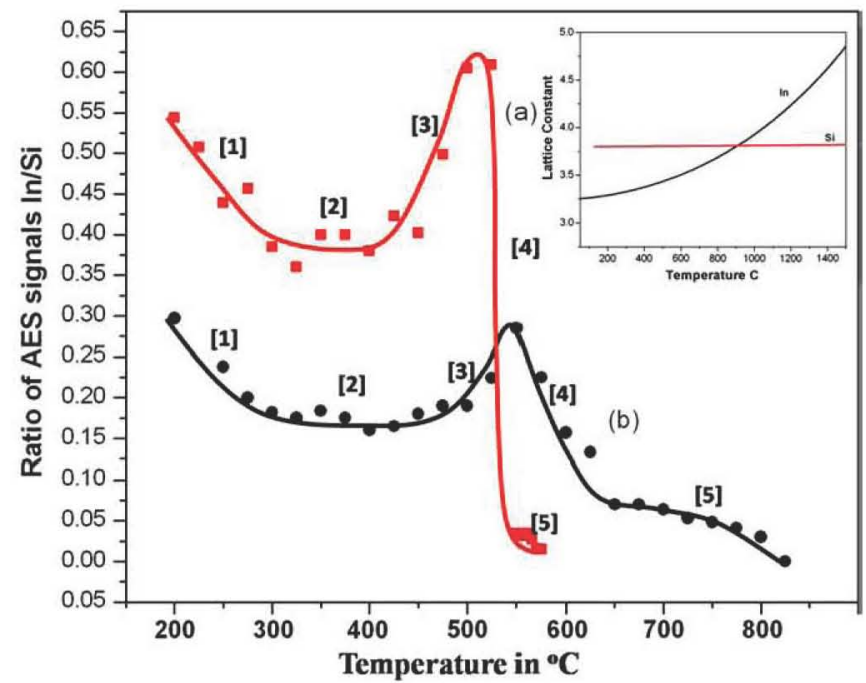

Fig. 5. The desorption curves of In on Si (111) (curve a) and Si (5 512 ) (curve b). The ratio of AES signal of In MNN (404 eV) to Si LVV $(92 \mathrm{eV})$ is plotted versus annealing temperature. The different regions on the two curves are marked in square brackets $[1,2]$ etc. The inset in the figure shows the variation of the Si and In lattice parameters with temperature. 

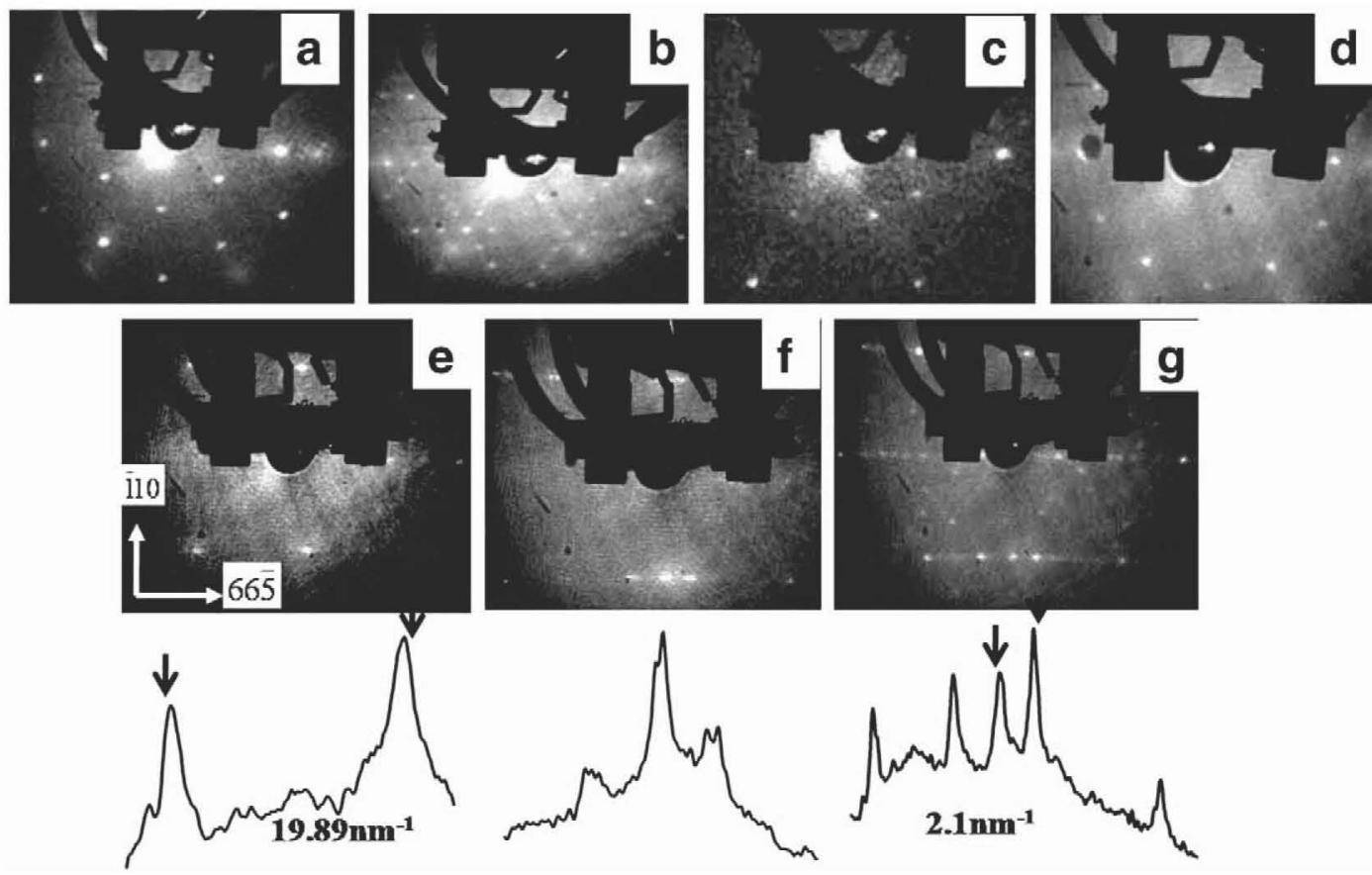

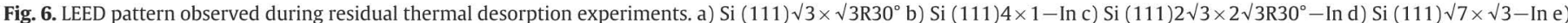

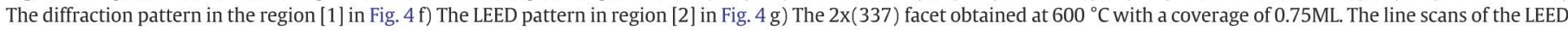
pattern for the Si ( 5512 ) surface taken along [66 5] direction are shown in the bottom panel.

surface we observe a few fractional order spots as shown in Fig. 6f. From the line scan we could not identify the phase to be of any particular facet as the number of reflections are limited and very weak. The rise in the intensity ratio in region [3] in Fig. 5, has the same temperature of about $450{ }^{\circ} \mathrm{C}$ on the two surfaces, and is attributed to the In islands converting into layers and consequently increasing the AES ratio. The activation energy for this layering is calculated to be $0.28 \mathrm{eV}$ on (111) and $0.40 \mathrm{eV}$ on (5 512 ) surfaces. The similarity in temperature on the two surfaces suggests presence of an energy barrier for layering. This could be the step-edge diffusion barrier which prevents the agglomerated clusters from layering. In this region at around $500{ }^{\circ} \mathrm{C}$ the line scan along the [66 5] direction shows (Fig. $2 \mathrm{~g}$ ) the presence of the $2 \times(113)$ facets on the surface. This is the same phase that we observed during the room temperature adsorption process at around 1.5ML. On the (111) surface we observe two superstructural phases viz. $\sqrt{ } 7 \mathrm{x} \sqrt{3}-$ In (Fig. 6d) and $4 \times 1-$ In (Fig. 6b) [30].

One of the important things to note here is that the stable layer above which clustering takes place is different for the two surfaces. In the case of $\mathrm{Si}(111)$ surface the agglomeration and layering takes place above the first flat 2ML of In, whereas for the Si (5 5 12) surface this happens on $1 \mathrm{ML}$ of In. Correlating with the LEED observations, the disappearance of the fractional order spots to $1 \times 1$ pattern on the $7 \times 7$ surface and the conversion of the $2 \times 225$ facet to $2 \times 113$ facets on (5 5 12) surface takes place at the above mentioned coverages, respectively. This can be related to the saturation of dangling bonds on the surfaces. On $\mathrm{Si}$ (111) surface, we have previously reported that the dangling bonds are saturated around 2ML of In adsorption [19], as inferred from the Si LVV (92 eV) peak excursion ratio. From a similar procedure we have estimated that on $\mathrm{Si}$ (5512) surface this takes place around $1 \mathrm{ML}$ of In adsorption, as the trenches may provide a more effective way of saturating the dangling bonds [19].

Beyond region [3] in Fig. 5, on both the surfaces, the intensity ratio falls because of the onset of desorption (sublimation) of Indium adatoms from the surface. The complete desorption for In on Si (111) surface takes place around $600^{\circ} \mathrm{C}$ and on the $\mathrm{Si}(5512)$ at about $850^{\circ} \mathrm{C}$. The onset temperature for desorption is different on the two surfaces $500{ }^{\circ} \mathrm{C}$ for $\mathrm{Si}(111)$ and $550{ }^{\circ} \mathrm{C}$ for $\mathrm{Si}(5512)$ surface. This temperature is higher for the Si (5 5 12) as compared to Si (111) surface, showing stronger bonding sites on the (5 5 12) surface owing to better coordination on this facetted surface. Another observation is that the desorption of In adatoms on the Si (5 5 12) surface takes place over a wide temperature range of $300{ }^{\circ} \mathrm{C}$ with a long tail, while on $\mathrm{Si}(111)$ it is around $50{ }^{\circ} \mathrm{C}$. This desorption at higher temperatures indicates the facetted nature of the (5 5 12) surface which provides stronger adsorption sites for the In adatoms. This region we have further divided into two subunits, region [4] and [5] shown in Fig. 5. On the Si (111) surface region [4] is very narrow and the $2 \sqrt{ } 3 \times 2 \sqrt{ } 3$ reconstruction (Fig. 6(c)) is observed in this region [31]. At around $600{ }^{\circ} \mathrm{C}$ on the $\mathrm{Si}(55$ 12) surface, the coverage is about $0.75 \mathrm{ML}$ and we observe an ordered LEED pattern. The intensity line scan taken along the [66 5] direction shows that the structure consists of $2 \times 337$ facets as seen in Fig. $6(\mathrm{~g})$ which has been reported for Au and other metals on Si (5 512 ) surface, to be made up of quasi 1D metal nanochain structures [26,31]. The interchain separation of these facets determined by measuring the separation between the LEED spots along the [66 5] direction is $3.2 \mathrm{~nm}$. The absence of the weak streak along the (110) direction indicates that the periodicity along this direction remains to be that of bulk structure. Here we find that the activation energy is high on the (111) surface, compared to the value $0.80 \mathrm{eV}$ on (5 512 ) owing to the fast desorption on the (111) surface with temperature.

On the (111) surface the final surface geometry after the sharp desorption is the $\mathrm{Si}(111) \sqrt{ } 3 \times \sqrt{ } 3 \mathrm{R} 30^{\circ}$ (Fig. 6a) - In surface, which appears in region [5], as indicated by the LEED pattern [32]. This is planar with bulk truncated silicon structure and is a stable surface as all the dangling bonds are satisfied [32]. Since the surface is chemically passivated, the In layers lying above are bonded with a specific energy to the substrate allowing a fast desorption in a narrow temperature window. In this region on the (5 512) surface, at around $700{ }^{\circ} \mathrm{C}$ we observe the $2 \times(225)$ from the LEED pattern, which is the same surface phase as the one observed during the RT adsorption at $0.5 \mathrm{ML}$. The difference in the value of the activation energies of $4.4 \mathrm{eV}$ on (111) and $0.54 \mathrm{eV}$ on (5 5 12) surface reflects the different facets from which the desorption of In atoms takes place. The dangling bond density on the (113) surface is less than that on the (337) which in 
turn is less than that on the (225) unit [15], and is manifested in the desorption sequence as well. The desorption region around $600{ }^{\circ} \mathrm{C}$ (region [4]) marks the formation of $2 x$ (337) facet which, owing to the lesser number of dangling bonds, shows comparatively weaker bonding and hence allows faster desorption as compared to the final desorption from the (225) facet (region [5]). Finally above $600^{\circ} \mathrm{C}$ on (111) and around $850^{\circ} \mathrm{C}$ on (55 12) surfaces, we retrieve the clean $7 \times 7$ and $2 \times 1$ reconstructions, respectively. A schematic of the desorption process for the Si (111) surface is shown in Fig. 4b assuming an initial coverage on the surface to be 3ML. For the Si (5 5 12) surface the overall picture remains the same, except that the stable layer is $1 \mathrm{ML}$ instead of $2 \mathrm{ML}$ in the (111) case.

Thus, qualitatively we observe a kind of retracing of the adsorption pathway during desorption with the sequence being $(225)+(337)-2 x$ (225) $-2 x(337)-2 x(113)$. The facet $2 x$ (337) could not be observed by LEED during the room temperature adsorption, due to weak fractional order spots. This reversibility in structural transformation on Si (5 5 12) surface and the clustering and layering on $\mathrm{Si}$ (111) surface show that the effect is geometric without any chemical interaction at the interface.

The clustering and layering evident in the adsorption and desorption process, is attributed to different origins. In case of the desorption process, the role of quantum size effect is less since the energy gain involved may be very less in comparison to other high temperature effects. If we look at the process thermodynamically, there is a huge lattice mismatch of about $14.8 \%$ and different thermal expansion coefficients [23]. Thus, an initial adsorbed ln clusters when subjected to annealing starts agglomerating on top of a wetting layer, in a short temperature. In the inset Fig. 5 we have plotted the bulk lattice parameters of silicon and indium as a function of temperature [33]. We find that with application of temperature there is a gradual reduction in the lattice mismatch which promotes layering of the In islands, due to reduced strain. Since the onset of the temperature for layering on both the surfaces is about $450{ }^{\circ} \mathrm{C}$, it is independent of the substrate surface geometry. Thus, the layering results from the relaxation of strain as well as the In atoms overcoming the step-edge diffusion barrier on islands.

\section{Conclusion}

This is a first report on the comparative study of the adsorption and desorption behavior of In adatoms on the low index $\mathrm{Si}(111)-7 \times 7$ surface and high index $\operatorname{Si}\left(\begin{array}{lll}5 & 5 & 12\end{array}\right)-2 \times 1$ surface. On both surfaces the room temperature adsorption show Stranski-Krastanov growth mode with island formation starting above 2ML. On Si (111) surface above 2ML we observe layering of 3D clusters which may be due to the quantum size effects operating at these low dimensional islands. The residual thermal desorption studies show the layering and clustering of In islands. We observe the differences in the stable layer above which agglomeration takes place for the two surfaces. The onset temperature of desorption and the temperature regime in which In completely desorbs from the surface is attributed to effective dangling bond saturation and strain relaxation, and the presence of a step-edge diffusion barrier. A comparison of the thermal expansion coefficient of In and Si at higher temperature shows that there is a reduction in the lattice mismatch between the adsorbate and substrate leading to lowering of strain which helps in the layering of In clusters. We have monitored the surface symmetry during adsorption and desorption, which shows different superstructural phases on $\mathrm{Si}$ (111). On the $\mathrm{Si}$ (5 512 ) surface we observe the formation of quasi $1 \mathrm{D}$ nanochain structures on the $2 \times 225,2 \times 113$ and $2 \times 337$ facets. These experiments using conventional techniques throw up interesting surface phenomena and encourage the probe of local effects using STM etc. To understand the role of quantum size confinement in the growth mode, the determination of step edge-barrier on the In islands, structure of the nanowires and the difference in stability of overlayers on the two surfaces demand more experiments and calculations in this direction.

\section{Acknowledgements}

The authors gratefully acknowledge Prof. C.N.R. Rao, JNCASR, Bangalore and Director, NPL, New Delhi for their constant encouragement and support. One of the authors (JK) acknowledges the UGC for a Junior Research Fellowship.

\section{References}

[1] E. Bauer, Z. Kristallogr. 110 (1958) 372.

[2] Zhenyu Zhang, Max G. Lagally, Science 276 (1997) 377.

[3] S.M. Shivaprasad, T. Abukava, H.W. Yeom, M. Nakamura, S. Suzuki, S. Sato, K. Sakamoto, T. Sakamoto, S. Kono, Surf. Sci. 344 (1995) L1245.

[4] S.M. Binz, M. Hupala, M.C. Tringides, Phys. Rev. B 78 (2008) 193407.

[5] Baris Unal, Alex Belianinov, P.A. Thiel, M.C. Tringides, Phys. Rev. B 81 (2010) 085411.

[6] D.M. Homes, J.G. Belk, J.L. Sudijono, J.H. Neeve, T.S. Jones, B.A. Joyce, J. Vac. Sci. Vac. Technol. 14 (1996) 849.

[7] J. Camarero, V. Cros, M.J. Capitan, J. Alvarez, S. Ferrer, M.A. Nino, J.E. Alvarez, S. Ferrer, M.A. Nino, J.E. Prieto, L. Gomez, J. Ferron, A.L. Vazquez de Parga, J.M. Gallego, J.J. de Miguel, R. Miranda, Appl. Phys. A Mater. 69 (1999) 553.

[8] J.V. Barth, et al., Nature 437 (2005) 29.

[9] Harumo Morikawa, C.C. Hwang, Hang Woong Yoem, Phys. Rev. B 81 (2010) 075401.

[10] Jin-Feng Jia, Xi. Liu, Jun-Zhong Wang, Jian-Long Li, X.S. Wang, Qi-Kun Xue, ZhiQiang Li, Zhenyu Zhang, S.B. Zhang, Phys. Rev. B 66 (2002) 165412.

[11] Mahesh Kumar, Vinod Kumar Paliwal, Amish G. Joshi, Govind, S.M. Shivaprasad, Surf. Sci. 596 (2005) 206.

[12] K.L. Men, Z.Q. Qiu, M.S. Altman, Phys. Rev. B 81 (2010) 045426.

[13] Ji Young Lee, Jun-Hyung Cho, Zhenyu Zhang, Phys. Rev. B 80 (2009) 155329.

[14] A.A. Baski, S.C. Erwin, L.J. Whitman, Science 269 (1995) 1556.

[15] Hidong Kim, Huiting Li, Yong-Zhe Zhu, J.R. Hahn, Jae M. Seo, Surf. Sci. 601 (2007) 1831.

[16] H. Ofner, S.L. Surnev, Y. Shapira, F.P. Netzer, Phys. Rev. B 48 (1993) 10940.

[17] J. Kraft, M.G. Ramsey, F.P. Netzer, Phys. Rev. B 55 (1995) 5384.

[18] J.J. Lander, J. Morrison, J. Appl. Phys. 36 (1965) 1706.

[19] Govind, K. Jithesh, Mahesh Kumar, S.M. Shivaprasad, J. Nanosci. Nanotech. 9 (1999) 5420.

[20] A. Ishizaka, Y. Shiraki, J. Electrochem. Soc. 133 (1986) 666.

[21] Y. Enta, S. Suzuki, S. Kono, Phys. Rev. B 39 (1989) 5524.

[22] K. Takayanagi, Y. Tanishiro, S. Takahashi, M. Takahashi, Surf. Sci. 164 (1985) 367.

[23] M.S. Finney, C. Norris, P.B. Howes, Surf. Sci. 277 (1992) 330.

[24] C. Argile, G.E. Rhead, Surf. Sci. Rep. 10 (1989) 277.

[25] S.S. Lee, N.D. Kim, C.G. Hwang, H.J. Song, J.W. Chung, Phys. Rev. B 66 (2002) 115317.

[26] A.A. Baski, K.M. Saoud, K.M. Jones, Appl. Surf. Sci. 182 (2001) 216.

[27] Michael C. Tringides, Mieczyslaw Jatochowski, Ernst Bauer, Phys. Today 51 (2007).

[28] D.K. Goswami, K. Battacharjee, B. Satpati, S. Roy, P.V. Satyam, B.N. Dev, Surf. Sci. 601 (2007) 603.

[29] Vinod Kumar Paliwal, A.G. Vedeshwar, S.M. Shivaprasad, Phys. Rev. B 66 (2002) 245404.

[30] K. Jithesh, Govind, V. Umesh, S.M. Waghmare, Shivaprasad 256 (2009) 348.

[31] Mahesh Kumar, Govind, V.K. Paliwal, A.G. Vedeshwar, Surf. Sci. 600 (2006) 2745.

[32] Demeter Tzeli, Ioannis D. Petsalakis, Giannoula Theodorakopoulos, J. Phys. Chem. C 113 (2009) 13924.

[33] E.V. Vernon, S. Weitroub, Proc. Phys. Soc. (London) B66 (1953) 887. 